\title{
Viable and Heat-Killed Probiotic Strains Improve Oral Immunity by Elevating the IgA Concentration in the Oral Mucosa
}

\author{
Wen-Yang Lin ${ }^{1}$ (I) Yi-Wei Kuo ${ }^{1}$. Ching-Wei Chen ${ }^{1}$ - Yu-Fen Huang ${ }^{1}$. Chen-Hung Hsu ${ }^{1}$. Jia-Hung Lin ${ }^{1}$. \\ Cheng-Ruei Liu ${ }^{1} \cdot$ Jui-Fen Chen ${ }^{1} \cdot \mathrm{Ko}-\mathrm{Chiang} \mathrm{Hsia}^{1} \cdot \mathrm{Hsieh}-\mathrm{Hsun} \mathrm{Ho}^{1}{ }^{10}$
}

Received: 6 October 2020 / Accepted: 9 June 2021 / Published online: 3 August 2021

(c) The Author(s) 2021

\begin{abstract}
Oral-nasal mucosal immunity plays a crucial role in protecting the body against bacterial and viral invasion. Safe probiotic products have been used to enhance human immunity and oral health. In this study, we verified the beneficial effects of mixed viable probiotic tablets, consisting of Lactobacillus salivarius subsp. salicinius AP-32, Bifidobacterium animalis subsp. lactis CP-9, and Lactobacillus paracasei ET-66, and heat-killed probiotic tablets, consisting of $L$. salivarius subsp. salicinius AP-32 and L. paracasei ET-66, on oral immunity among 45 healthy participants. Participants were randomly divided into viable probiotic, heat-killed probiotic, and placebo groups. The administration of treatment lasted for 4 weeks. Saliva samples were collected at Weeks 0, 2, 4, and 6, and Lactobacillus, Bifidobacterium and Streptococcus mutans populations and IgA concentration were measured. IgA concentrations, levels of TGF-beta and IL-10 in PBMCs cells were quantified by ELISA method. Results showed that salivary IgA levels were significantly increased on administration of both the viable $(119.30 \pm 12.63 \%, * * * P<0.001)$ and heat-killed $(116.78 \pm 12.28 \%, * * * P<0.001)$ probiotics for 4 weeks. Among three probiotic strains, AP-32 would effectively increase the levels of TGF-beta and IL-10 in PBMCs. The oral pathogen Streptococcus mutans was significantly reduced on viable probiotic tablet administration $(49.60 \pm 31.01 \%$, ***P<0.001). The in vitro antibacterial test confirmed that viable probiotics effectively limited the survival rate of oral pathogens. Thus, this clinical pilot study demonstrated that oral probiotic tablets both in viable form or heat-killed form could exert beneficial effects on oral immunity via IL-10, TGB-beta mediated IgA secretion. The effective dosage of viable probiotic content in the oral tablet was $10^{9} \mathrm{CFUs} / \mathrm{g}$ and the heat-killed oral tablet was $1 \times 10^{10} \mathrm{cells} / \mathrm{g}$.
\end{abstract}

\section{Introduction}

The oral-nasal mucosa, the gateway for contacting inhaled antigens, is the frontline defense of the human immune system $[1,2]$. Usually, pathogenic viruses or bacteria invade mucosal surfaces of the oral-nasal cavity by fighting against the local immune system and the healthy microbiota [3]. Inflammation and symptoms, including cough, sore throat, runny nose, and fever, develop due to the immune responses against the invasive pathogenic viruses and bacteria [4].

Additionally, immunoglobulin A ( $\operatorname{Ig} \mathrm{A})$ is the main immune factor in saliva and regulates homeostasis of the oral microbiota [5]. Several studies have reported the ability

Hsieh-Hsun Ho

sam.ho@bioflag.com.tw

1 Department of Research and Design, Bioflag Biotech Co., Ltd., 4F.C2, No. 17, Guoji Rd, Xinshi Dist, Tainan City 744, Taiwan of secretory $\operatorname{IgA}$ to protect the body from infection by viral pathogens, including respiratory syncytial virus [6], rotavirus [7], and influenza virus [7]. Secretory $\operatorname{Ig} A$ is functioned to protect mucosal epithelia. Nicolas Millet et al. discovered that mucosal IgA can prevent commensal Candida albicans dysbiosis in the oral cavity [8]. It also prevents aggregation of the oral pathogen Streptococcus mutans, which causes dental plaque [9].

Besides, researchers discovered that the oral microbiota and its metabolites may locally influence immunity at the oral barrier [10]. Previous studies demonstrated the effectiveness of probiotics on the elevating the $\operatorname{IgA}$ concentration in the oral mucosa [11]. Ericson et al. demonstrated that chewing gum contained Lactobacillus reuteri participated in the adaptive immune response of healthy subjects by increasing the salivary IgA levels [12]. In human, the immune cytokines TGF-beta and IL-10 are correlated to the production of $\operatorname{Ig} \mathrm{A}$ [13]. 
However, whether oral microbiota elevated secretory IgA level through cytokines TGF-beta and IL-10 are still unclear.

Furthermore, the oral pathogens including S. mutans, $P$. gingivalis, $F$. nucleatum subsp. polymorphum, and $A$. actinomycetemcomitans showed high correlation with periodontal disease and bad breath [14]. Probiotic product could be a potential alternative therapy to periodontitis by inhibiting oral pathogens Fusobacterium nucleatum, but Porphyromonas gingivalis and Aggregatibacter actinomycetemcomitans showed no significant decrease [15]. Probiotic supplementation can also prevent pathogenic infections [16]. It has been reported that key species of the microbiota limited the growth of pathogenic Streptococcus pneumonia [17]. Thus, maintaining a balanced microbial ecosystem via the uptake of probiotic strains may be an alternative way to regulate oral immunity and enhance oral health.

Previous findings on the inhibition of oral pathogens by probiotic strains have revealed that Lactobacillus salivarius subsp. salicinius AP-32, Bifidobacterium animalis subsp. lactis CP-9, and Lactobacillus paracasei ET-66 exert excellent antibacterial effects in vitro; however, not by the mechanism of increasing $\mathrm{H}_{2} \mathrm{O}_{2}$ levels [18]. The major strategies for probiotic strains to limit oral pathogenic growth were co-aggregating pathogens in oral, producing biosurfactants, to change oral environment, generating antimicrobial substances [19]. Additionally, an in vitro study showed that $L$. salicinius AP-32 mediated inflammatory cytokine [interleukin (IL)-8, IL-10] expression in human epithelial cells [20].

Therefore, in this study, we verified whether mixed viable probiotic tablets, consisting of $L$. salivarius subsp. salicinius AP-32, B. animalis subsp. lactis CP-9, and L. paracasei ET-66, and heat-killed probiotic tablets, consisting of $L$. salivarius subsp. salicinius AP-32 and L. paracasei ET-66, improved immune function via TGF-beta, IL-10 or elevated the level of salivary IgA concentration. The anti-bacterial function of probiotic product uptake in the oral cavity was tested.

\section{Material and Methods}

\section{Participants}

In total, 45 healthy participants aged $20-40$ years were recruited and randomly divided into three subgroups: the viable probiotic oral tablet, heat-killed probiotic oral tablet, and placebo groups. Each group consisted of 15 participants. Participants with smoking and betel nut eating habits were excluded. Oral tablets were blindly assigned to the participants. All participants took the oral tablets thrice a day (morning, noontime, and evening) for 4 weeks. Saliva samples $(2 \mathrm{~mL})$ were collected to measure IgA concentration and microorganism populations at Weeks 0,2 , 4, and
6. Informed consent was obtained from all participants included in this study. The protocol was approved by the Institutional Review Board of Chung Shan Medical University, Taiwan (CS19052).

\section{Viable Probiotic Oral Tablets}

The viable probiotic oral tablet $(1 \mathrm{~g})$ contained three viable probiotic strains, $L$. salivarius subsp. salicinius AP-32, $B$. animalis subsp. lactis CP-9, and L. paracasei ET-66. We mixed $0.01 \mathrm{~g}$ of $10^{11}$ colony formation units (CFUs) single probiotic in oral tablet. Each single probiotic strain was measured around $0.33 * 10^{9} \mathrm{CFUs} / \mathrm{g}$. The total probiotic content in the oral tablet was $10^{9}$ colony CFUs/g. The active dosage of probiotic oral tablet were following previous published works [18]. All probiotic strains were obtained from the laboratory of Bioflag Biotech Co., Ltd. (Tainan, Taiwan). L. salivarius subsp. salicinius AP-32 was isolated from healthy human gut and deposited in Food Industry Research and Development Institute, Taiwan (ID: BCRC 910437) and in Wuhan university, China (ID: CCTCC-M2011127); $B$. animalis subsp. lactis $\mathrm{CP}-9$ was isolated from healthy human breast milk and deposited in Food Industry Research and Development Institute, Taiwan (ID: BCRC 910645) and in Wuhan university (ID: CCTCC-M2014588); L. paracasei ET-66 was isolated from healthy human breast milk and deposited in Food Industry Research and Development Institute, Taiwan (ID: BCRC 910753) and in China General Microbiological Culture Collection Center, Beijing, China (ID: CGMCC-13514).

Lactobacillus. salivarius subsp. salicinius AP-32 and L. paracasei ET-66 were cultured on de Man, Rogosa, and Sharpe (MRS) agar plates (110,660, Merck, Darmstadt, Germany) and incubated under facultative anaerobic conditions at $37{ }^{\circ} \mathrm{C}$ for 48 h. B. animalis subsp. lactis CP-9 was cultured on MRS agar supplemented with $0.05 \%$ cysteine and anaerobically incubated at $37{ }^{\circ} \mathrm{C}$ for $48 \mathrm{~h}$. Counting colonies was performed for quantifying bacterial $\mathrm{CFU}$ after culturing probiotic strains [18].

\section{Heat-killed Probiotic Oral Tablets}

The heat-killed probiotic oral tablets $(1 \mathrm{~g})$ consisted of inactivated L. salivarius subsp. salicinius AP-32 and L. paracasei ET-66. The viable strains were cultured and incubated, as previously described. Then, AP-32 and ET-66 were inactivated by incubating the viable strains in MRS media at $100{ }^{\circ} \mathrm{C}$ for $1 \mathrm{~h}$. The probiotic content in the oral tablet was $1 \times 10^{10} \mathrm{cells} / \mathrm{g}$. The active dosage of heat-killed probiotic oral tablet were following previous published in vitro testing [11]. All experimental procedures were performed in the laboratory of Bioflag Biotech Co., Ltd. (Tainan, Taiwan). 


\section{Measurement of the Lactobacillus} and Bifidobacterium Populations in Saliva Samples

Saliva samples $(2 \mathrm{~mL})$ were collected from each participant at Weeks $0,2,4$, and 6 . The saliva samples $(100 \mu \mathrm{L})$ were serially diluted and added to MRS plates. To measure Lactobacillus populations, Lactobacillus was cultured and incubated on the MRS agar plates under facultative anaerobic conditions at $37{ }^{\circ} \mathrm{C}$ for $48 \mathrm{~h}$. To measure Bifidobacterium populations, Bifidobacterium was cultured and incubated anaerobically on MRS agar supplemented with $0.05 \%$ cysteine at $37{ }^{\circ} \mathrm{C}$ for $48 \mathrm{~h}$. Counting colonies was performed for quantifying bacterial CFU after culturing probiotic strains. The Lactobacillus and Bifidobacterium population rates were normalized to the probiotic population number at Week 0 . The population rate of Lactobacillus and Bifidobacterium collected at Week 0 was considered $100 \%$. The experimental method followed previous published works [18].

\section{Measurement of the IgA Concentration in the Oral Mucosa}

Saliva samples $(2 \mathrm{~mL})$ were collected from each participant at Weeks $0,2,4$, and 6 and maintained at $-20{ }^{\circ} \mathrm{C}$. The $\operatorname{IgA}$ Human Uncoated ELISA Kit (Catalog \# 88-50600-88; Invitrogen, Thermo Fisher Scientific, San Jose, CA, USA) was used to analyze the IgA concentration. Firstly, we coated anti-human IgA antibodies on 96-well plate. Salivary $\operatorname{IgA}$ present in the sample or standard were added to the microwell, then HRP-conjugated anti-human IgA antibody was added at room temperature for $1 \mathrm{~h}$. Pipetting TMB Substrate to each well for $30 \mathrm{~min}$. Finally, the IgA concertation was measured on a spectro-photometer at $450 \mathrm{~nm}$. All experimental procedure was performed in triplicate, according to manufacturer's instructions. The concentration rate of $\operatorname{IgA}$ collected at Week 0 was considered $100 \%$. The experimental method followed previous published works [18].

\section{Measurement of the TGF-Beta and IL-10 Concentration in PBMC}

The testing of probiotic induced elevation of TGF-beta and IL-10 level was following the previous study [20]. The peripheral blood mononuclear cells (PBMCs) were obtained from blood samples of healthy donors (Tainan Blood Center, Taiwan Blood Services Foundation). Centrifuging whole blood through a Ficoll-Hypaque gradient (Pharmacia, Sweden), and the light-density fraction from the $42.5 \%-50 \%$ interface was eluted. Seeding $4 * 10^{5}$ cell extracted PBMCs cells in $100 \mu \mathrm{R}$ RPMI-1640 with 1\% FBS (Sigma Chemical Co., St. Louis, MO, USA) to each well of 96 well plate. Then adding $5 * 10^{5} \mathrm{cfu} / 20 \mu \mathrm{l}$ probiotic treatment to each
PBMCs seeded well (cells: probiotic strains $=1: 10$ ). The positive controls were adding $10 \mu \mathrm{g} / \mathrm{ml}$ Phytohaemagglutinin (PHA) (Sigma-Aldrich, Munich, Germany) to PBMCs seeded wells for the production of TGF- $\beta$, IL-10 respectively [21]. Co-culturing probiotics with PBMCs for $48 \mathrm{~h}$. Measuring supernatant containing cytokines IL-10 and TGF- $\beta$ by enzyme-linked immunosorbent assay (ELISA) analysis (Thermo Scientific, Carlsbad, CA, USA). All experiments were measured and followed the commercial protocol at least in triplicate.

\section{Evaluation of the Antibacterial Function in Oral Cavity}

Streptococcus mutans is a common oral health threat, which caused dental caries by forming plague or biofilms [22]. Streptococcus mutans samples was collected from the swabs, by which we collected the $S$. mutans on supragingival part, gingivae and oral mucosa, at Weeks $0,2,4$, and 6 . S. mutans was maintained in tryptic soy broth (TSB, $5 \mathrm{~mL}$ ) supplemented with $50 \%$ glycerol. The $S$. mutans medium was serially diluted and added to the Mitis Salivarius-Bacitracin Agar (MSBA, $0.2 \mathrm{U} / \mathrm{mL}$ ) plate, which was incubated at $37{ }^{\circ} \mathrm{C}$ for $2 \mathrm{~d}$. Finally, the number of $S$. mutans colonies in each participant sample was counted to calculate pathogenic survival rates; the survival rate of $S$. mutans collected at Week 0 was considered $100 \%$. The experimental method followed previous published works [18].

\section{In Vitro Antibacterial Activity of the Oral Tablets}

Oral tablets $(1 \mathrm{~g})$ that contained viable (AP-32, CP-9, and ET-66; $>10^{9}$ CFUs) and heat-killed (AP-32 and ET-66, $>10{ }^{10}$ CFUs) probiotic strains were dissolved in TSB and brain heart infusion medium, respectively, to obtain a final concentration of $0.1 \mathrm{~g} / \mathrm{mL}$. Only the medium was used for the blank group, whereas tablets $(1 \mathrm{~g})$ without any probiotic components were dissolved in the respective media for the placebo group. Then, oral pathogens $\left(10^{6} \mathrm{CFUs}\right)$ were introduced into the tablet solutions and co-incubated at $37^{\circ} \mathrm{C}$ for 2 (S. mutans) or 3 (Porphyromonas gingivalis, Fusobacterium nucleatum subsp. polymorphum, and Aggregatibacter actinomycetemcomitans) d. Pathogens were obtained from the BCRC. The oral pathogen inhibition rate (\%) was calculated as follows: ( $\left.\mathrm{CFU}_{\text {blank control }}-\mathrm{CFU}_{\text {experimental group }}\right) /$ $\mathrm{CFU}_{\text {blank control. }}$ The experimental method followed previous published testing [11].

\section{Statistical Analysis}

GraphPad Prism software was used for statistical analysis of two-tailed t-test results. Data were presented as the mean \pm standard deviation (SD) or the mean of the results of 
two or three independent experiments. Statistical differences were considered significant at $P$ value $<0.05$.

\section{Results}

\section{Probiotic Tablets Increased the Bifidobacterium Population in the Oral Cavity}

The Bifidobacterium population was stably elevated in the oral cavity after probiotic tablet administration. The bacterial populations after treatments were normalized by bacterial populations before treatments (Population rate $\%=$ post-treatment $\mathrm{CFU} /$ pre-treatment $\mathrm{CFU})$. In the viable probiotic group, the population rate significantly increased to $244.14 \pm 164.96 \%$ at Week $2(* * P<0.01$, Fig. $1 \mathrm{a})$ and $438.41 \pm 308.58$ at Week $4(* * * P<0.001)$, compared to Week 0 . The Bifidobacterium population rate remained high at Week 6 (no administration of viable probiotic tablets for 2 weeks after Week 4, 550.16 $\pm 448.19 \%$, *** $P<0.001)$, compared to Week 0 . The Bifidobacterium population number was significantly different between the viable probiotic and placebo groups at Week $6(* * P<0.01)$.

In the heat-killed probiotic group, the Bifidobacterium population was increased at Weeks 2 (149.92 $\pm 60.47 \%$,

a

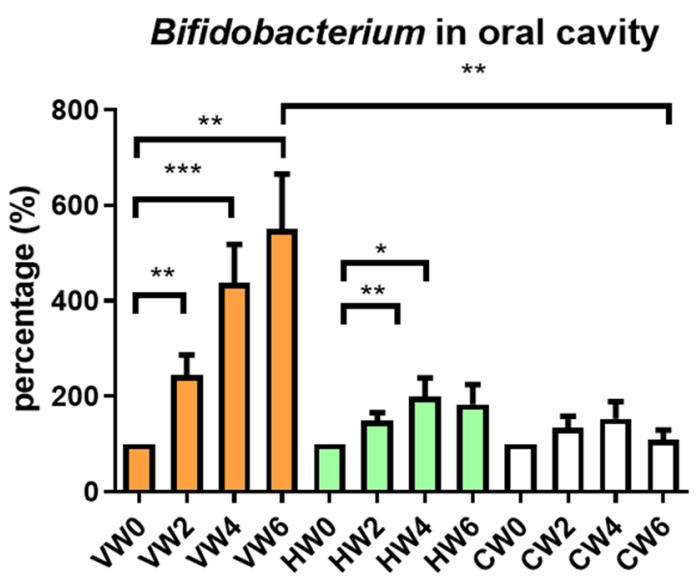

$* * P<0.01)$ and $4(199.87 \pm 148.64 \%, * P<0.05)$, but was decreased at Week 6 after administration of the heat-killed probiotic tablets was stopped for 2 weeks (182.98 $\pm 163.73 \%$, Fig. 1a).

\section{Probiotic Tablets Increased the Lactobacillus Population in the Oral Cavity}

The change in the oral Lactobacillus population due to probiotic tablet administration revealed a pattern similar to that followed by the change in the oral Bifidobacterium population. The bacterial populations after treatments were normalized by bacterial populations before treatments. In the viable probiotic group, the Lactobacillus population rate significantly increased to $447.74 \pm 337.89 \%$ at Week $2(* * P<0.01$, Fig. 1b) and $982.27 \pm 726.66 \%$ at Week $4(* * * P<0.001)$, compared to Week 0 . The Lactobacillus population rate was higher at Week 6 (no administration of viable probiotic tablets for 2 weeks after Week 4, 727.57 $\pm 539.76 \%$, $* * * P<0.001)$ than Week 0 . The Lactobacillus population number was significant different between the viable probiotic and placebo groups at Week $6(* * * P<0.001)$. In the heat-killed probiotic group, the Lactobacillus population was increased at Weeks $2(256.66 \pm 183.78 \%, * * P<0.01)$ and $4(312.85 \pm 279.71 \%, * P<0.05)$. Additionally, the

b

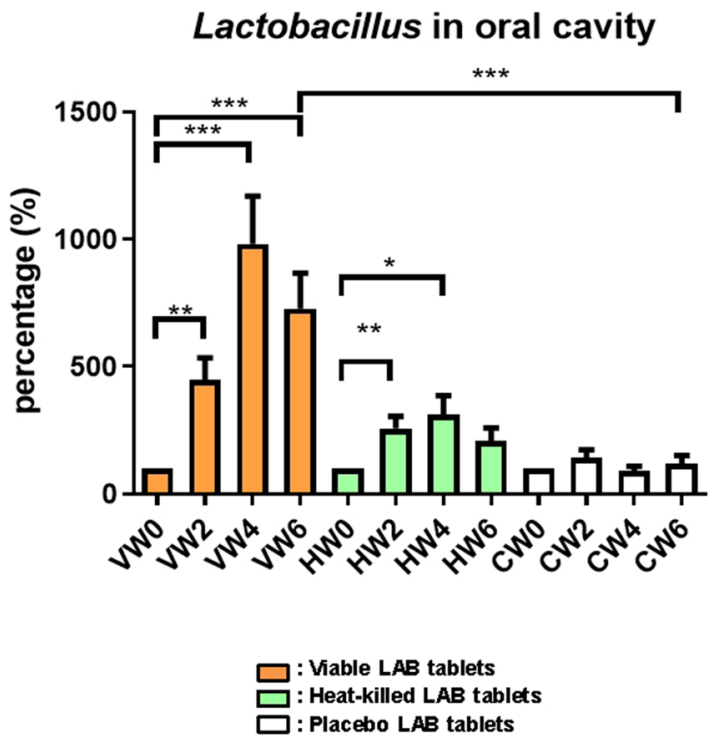

tions on the oral mucosal surfaces. $V$ viable probiotic tablets; $H$ heatkilled inactivated probiotic tablets; $C$ placebo tablets (control); and $L A B$ lactic acid bacteria. Collection and analysis of salivary samples at Weeks 0, 2, 4, and 6 were performed. Data were presented as the mean \pm standard deviation (SD). Two-tailed $t$-test was used to analyzed statistical difference. Statistical significances were marked with $* P<0.05, * * P<0.01, * * * P<0.001$ 
Lactobacillus number remained but showed no significant difference at Week 6 after administration of heat-killed probiotics was stopped for 2 weeks $(208.00 \pm 199.50 \%$, Fig. 1b).

\section{Probiotic Tablets Containing AP-32, ET-66, and CP-9 Significantly Elevated the IgA Concentration in the Oral Mucosa}

As probiotic tablet (viable or heat-killed) consumption has been proven to elevate Bifidobacterium and Lactobacillus levels in the oral cavity, we further analyzed the salivary IgA concentration changes, which occurred due to the uptake of probiotic products. The IgA levels after treatments were normalized by $\operatorname{IgA}$ levels before treatments. In the viable probiotic group, the salivary $\operatorname{Ig}$ A concentration slightly increased to $103.24 \pm 8.10 \%$ at Week 2 (Fig. 2) and $104.81 \pm 13.26$ at Week 4, but without statistically significant differences. However, the salivary IgA concentration in the viable probiotic group, compared to in the placebo group, was significantly increased to $119.30 \pm 12.63 \%$ at Week 6 (no administration of viable probiotic tablets for 2 weeks after Week 4), compared to Week $0(* * * P<0.001)$. Furthermore, in the heat-killed probiotic group, the salivary IgA concentration

\section{$\lg A$}
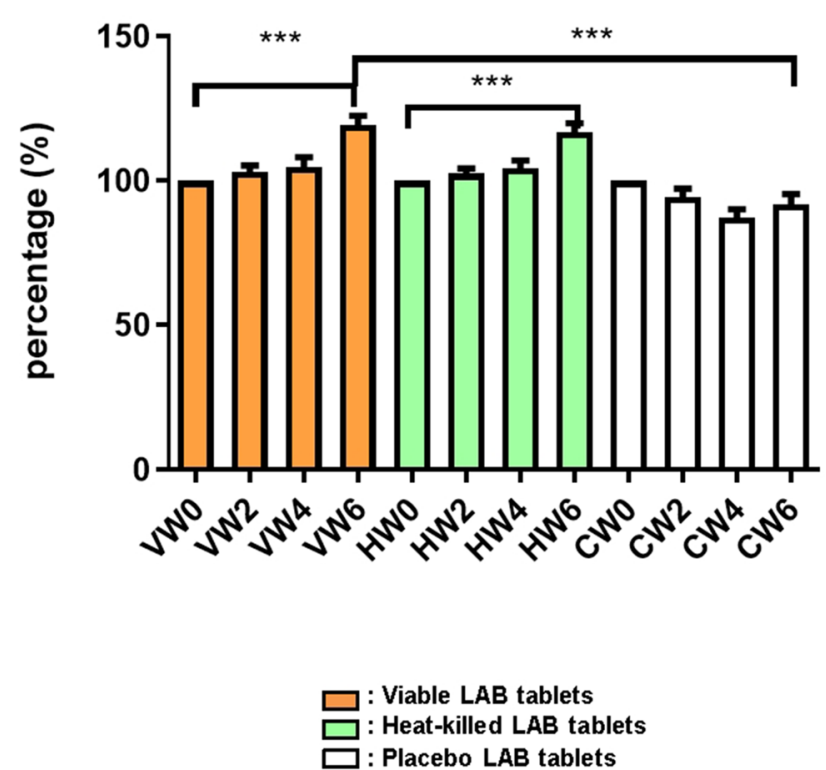

Fig. 2 Immunoglobulin A (IgA) concentration in the saliva samples. $V$ viable probiotic tablets; $H$ heat-killed inactivated probiotic tablets; $C$ placebo tablets (control); and $L A B$ lactic acid bacteria. Collection and analysis of salivary samples at Weeks $0,2,4$, and 6 were performed. Data were presented as the mean \pm standard deviation (SD). Two-tailed $t$-test was used to analyzed statistical difference. Statistical significances were marked with $* P<0.05$, $* * P<0.01$, $* * * P<0.001$ was slightly increased at Weeks $2(102.51 \pm 7.20 \%)$ and $4(104.35 \pm 10.27 \%)$. The saliva IgA concentration was increased at Week $6(116.78 \pm 12.28 \%, * * * P<0.001$, Fig. 2), compared to Week 0.

\section{Viable Strains Increased IL-10 and TGF-Beta Levels in PBMCs}

The positive control of $10 \mu \mathrm{g} / \mathrm{ml}$ Phytohaemagglutinin (PHA) was successfully elevated the levels of IL-10 $(58.2 \pm 7.9 \mathrm{pg} / \mathrm{ml}, * * * P<0.001$, Fig. $3 \mathrm{a})$ and TGF- $\beta$ $(53.4 \pm 8 \mathrm{pg} / \mathrm{ml}, * * * P<0.001$, Fig. 3b) in PBMCs by comparing to the negative control (PBMCs treated with medium only). Viable strains of CP-9, AP-32 and ET-66 significantly increased the levels of IL-10 in PBMCs to $84.2 \pm 9 \mathrm{pg} / \mathrm{ml}$ $(* * * P<0.001, \# \# P<0.01), 303.1 \pm 170.9 \mathrm{pg} / \mathrm{ml}(* P<0.05$, $\# \# P<0.01)$ and $98.2 \pm 17.5 \mathrm{pg} / \mathrm{ml}(* * * P<0.01$, \#\#P<0.01), respectively. Symbol *indicated significant difference by comparing to the medium treated control; symbol \#indicated significant difference by comparing to the PHA control (Fig. 3a).

Besides, viable strains of CP-9, AP-32 and ET-66 significantly increased the levels of TGF-beta in PBMCs to $23.2 \pm 4.7 \mathrm{pg} / \mathrm{ml}(* * * P<0.001), 130.9 \pm 16 \mathrm{pg} /$ $\mathrm{ml}(* * * P<0.001$, \#\#\#P<0.001) and $31.4 \pm 5.5 \mathrm{pg} / \mathrm{ml}$ $(* * * P<0.01)$, respectively (Fig. $3 b)$.

\section{Probiotic Tablets Containing AP-32, ET-66, and CP-9 Prohibited the S. mutans Population in the Saliva}

Elevated Bifidobacterium and Lactobacillus populations and increased salivary IgA concentration might contribute to the fight against oral pathogenic microorganisms. In the subsequent experiment, we tested the alteration in the oral pathogen $S$. mutans population due to probiotic intervention. The bacterial populations after treatments were normalized by bacterial populations before treatments. In the viable probiotic group, the $S$. mutans population was significantly reduced at Weeks $2(53.55 \pm 31.60 \%, * * * P<0.001)$, $4(49.60 \pm 31.01 \%, * * * P<0.001)$, and $6(55.19 \pm 42.72 \%$, $* * \mathrm{P}<0.01$, Fig. 4). At week 6, S. mutans was significantly inhibited in the viable probiotic group $(55.19 \pm 42.72 \%$, $* * * P<0.001)$, compared to in the placebo group. Furthermore, in the heat-killed probiotic group, the $S$. mutans population was slightly decreased at Week $2(85.81 \pm 49.87 \%)$ and significantly reduced at Week $4(58.35 \pm 33.17 \%$, $* * * P<0.001)$. The $S$. mutans population level in the heatkilled probiotic group $(202.81 \pm 167.99 \%)$ reverted to that in the placebo group $(225.05 \pm 127.01 \%)$ at Week 6 (no administration of heat-killed probiotic tablets for 2 weeks from Week 4) (Fig. 4). 
a

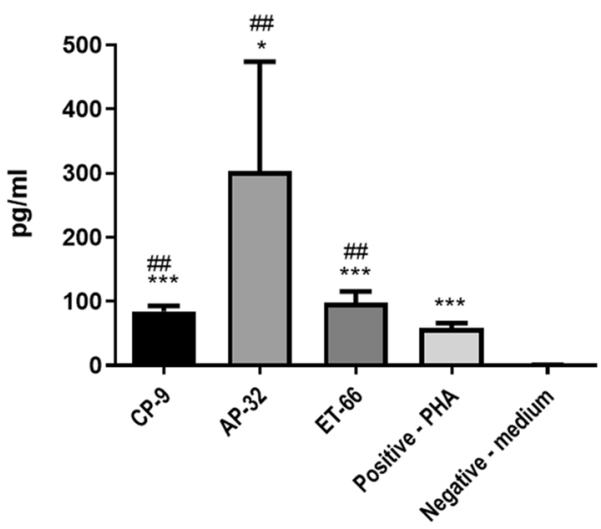

Fig. 3 Viable strains increased IL-10, TGF-beta, levels in PBMCs. The level of a IL-10 and b TGF-beta in PBMCs were tested by treating probiotic strains. Adding $5 * 10^{5} \mathrm{cfu} / 20 \mu \mathrm{l}$ viable strains of CP-9, AP-32 and ET-66 to PMBCs $10 \mu \mathrm{g} / \mathrm{ml}$. Phytohaemagglutinin (PHA) was used as positive control. The negative control was PBMCs treated with RMPI-1640 medium only. Triplicate tests were per-

\section{S. mutans bioburden in oral cavity}
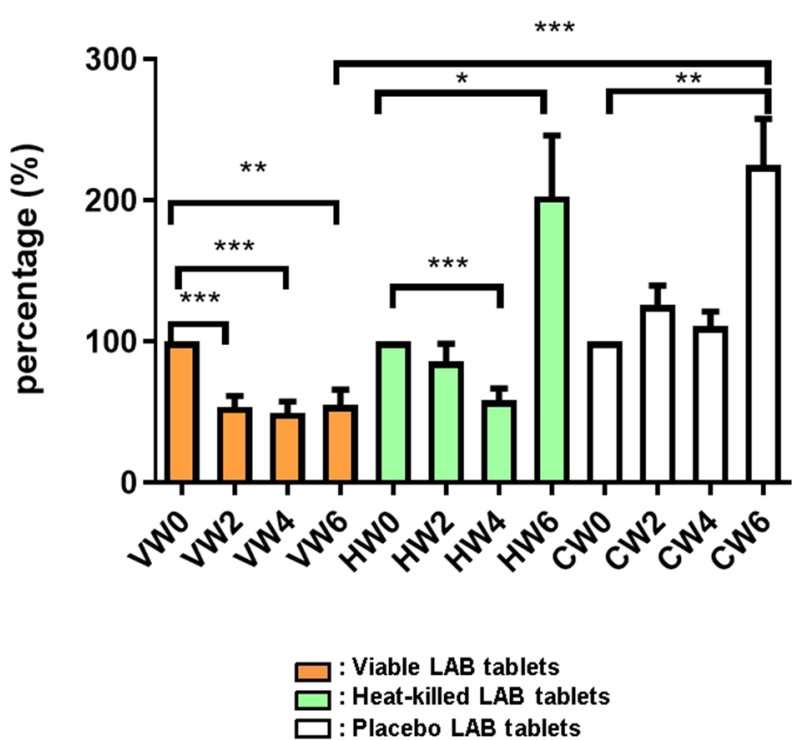

Fig. 4 Measurement of pathogenic Streptococcus mutans populations on the oral mucosal surfaces. $V$ viable probiotic tablets; $H$ heatkilled inactivated probiotic tablets; $C$ placebo tablets (control); and $L A B$ lactic acid bacteria. Collection and analysis of salivary samples at Weeks 0, 2, 4, and 6 were performed. Data were presented as the mean \pm standard deviation (SD). Two-tailed $t$-test was used to analyzed statistical difference. Statistical significances were marked with $* P<0.05, * * P<0.01, * * * P<0.001$ b

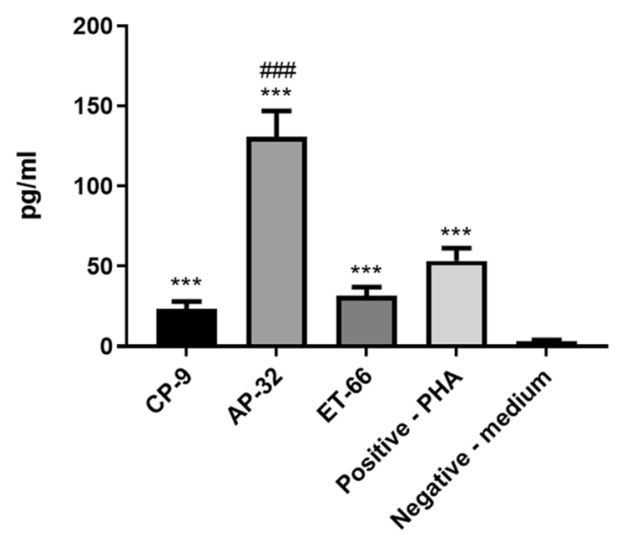

formed. Data were presented as the mean \pm standard deviation (SD). Two-tailed $t$-test was used to analyzed statistical difference. Statistical significances were marked with $P<0.05, P<0.01, P<0.001$. Symbol *indicated significant difference by comparing to the medium treated control; symbol \#indicated significant difference by comparing to the PHA control

\section{Confirmation of the Ability of Probiotic Tablets to Inhibit Oral Pathogen Growth by an In Vitro Test}

The in vitro study revealed that viable probiotic tablets significantly inhibited the survival rate of oral pathogens (Fig. 5a). The bacterial populations after treatments were normalized by bacterial populations before treatments. The survival rate of $S$. mutans, $P$. gingivalis, $F$. nucleatum, and A. actinomycetemcomitans in the viable probiotic group, compared to in the placebo group, was decreased to $4.23 \%$ $(* * * P<0.001$, placebo $=91 \%), 8.64 \%(* * * P<0.001$, placebo $=89 \%), 5.77 \%(* * P<0.01$, placebo $=98 \%)$, and $6.23 \%(* * P<0.01$, placebo $=87 \%)$, respectively. However, the heat-killed probiotic tablet exerted weaker antibacterial effects than the viable probiotic tablet (Fig. 5b). The survival rate of $S$. mutans, $P$. gingivalis, $F$. nucleatum, and $A$. actinomycetemcomitans in the heat-killed probiotic group, compared to in the placebo group, was slightly decreased without statistical significance $(82 \%$, placebo $=91 \%)$, significantly reduced to $53 \%(* P<0.05$, placebo $=89 \%)$, not inhibited $(96.88 \%$, placebo $=98 \%)$, and significantly reduced to $80 \%(* * P<0.01$, placebo $=87 \%)$, respectively.

\section{Discussion}

Comparing to the placebo group, the oral populations of Lactobacillus and Bifidobacterium would significantly increase by feeding viable probiotic tablet or heat-killed probiotic tablet for 4 weeks. Additionally, the 

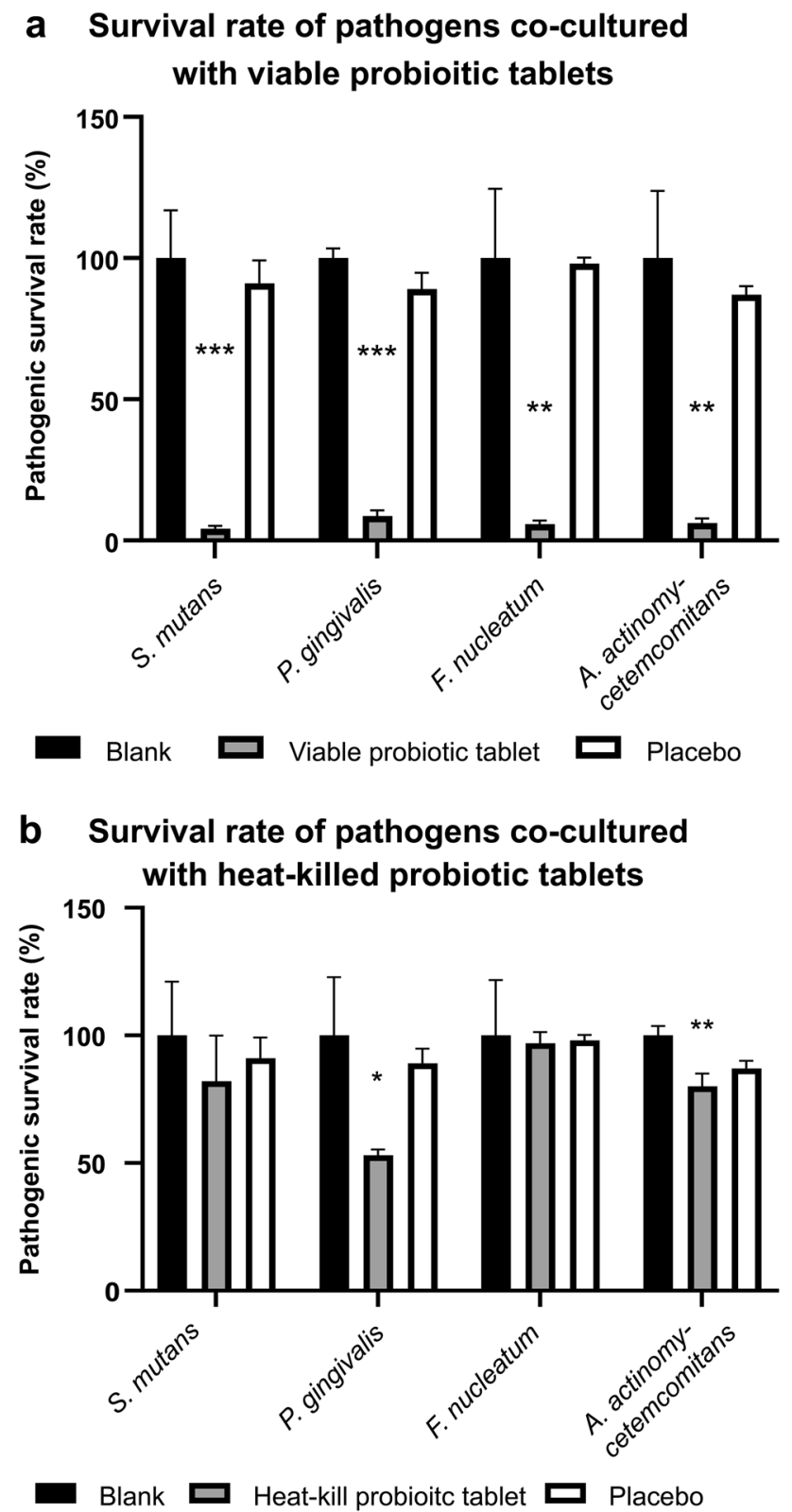

Fig. 5 Confirmation of the ability of probiotic tablets to inhibit oral pathogen growth by an in vitro test. The ability of $\mathbf{a}$ viable and $\mathbf{b}$ heat-killed probiotic tablets to inhibit the survival rate of oral pathogens, including Streptococcus mutans, Porphyromonas gingivalis, Fusobacterium nucleatum, and Aggregatibacter actinomycetemcomitans, was validated. Blank group, medium only; placebo group, tablets $(1 \mathrm{~g})$ without any probiotic components. Triplicate tests were performed. Data were presented as the mean \pm standard deviation (SD). Two-tailed $t$-test was used to analyzed statistical difference. Statistical significances were marked with $* P<0.05, * * P<0.01$, *** $P<0.001$

Lactobacillus and Bifidobacterium number remained significant high level for 2 weeks after suspension the consumption of viable probiotics consumption (Fig. 1a and b). Similarly, Camila Casuccio Almeida et al. demonstrated that four-week administration of a probiotic treatment could persist ameliorate symptoms in lactose-intolerant patients for at least 3 months after stopping probiotic consumption [23]. However, persisted effect of probiotic in long-term treatment should be evaluated in the future.

Additionally, salivary IgA concentration was significantly increased on administration of viable and heatkilled probiotic tablets. Both heat-killed and viable probiotic groups elevated salivary IgA by administrating oral tablets for 4 weeks, but without showing statistical difference to the placebo. The IgA concentration in heat-killed and viable probiotic groups showed significant increase at Week 6. However, the mechanism of IgA elevation after ceasing the probiotic administration for 2 weeks should be investigated further (Fig. 2) Braathen et al. reported similar results on the immune-modulating role of probiotic strains in the oral cavity [24]. Additionally, probiotic strains have been reported to increase fecal Ig A concentration significantly in sows and piglets [25]. However, studies on IgA-alleviating symptoms in upper airway infections remain insufficient. The detailed molecular mechanism of inhibition and prevention of upper airway infections by probiotic strains CP-9, AP-32, and ET-66 could be investigated in future studies.

At present study, viable strains CP-9, AP-32, and ET-66 would elevate the level of IL-10, TFG-beta in PBMCs cells. AP-32 showed effective ability in increasing IL-10, TFG-beta in PBMCs by comparing to PHA positive control (Fig. 3a and b). It presumed that viable strains CP-9, AP-32, and ET-66 might induce B-cell secreting IL-10 and TFG-beta to increase salivary IgA level. However, the correlation between salivary IL-10, TFG-beta and salivary IgA should be tested further [26]. Besides, whether heat-killed probiotic elevated IgA via inducing IL-10 and TFG-beta are still unclear. An in vitro test indicated that heat-killed AP-32 promoted interferon- $\gamma$ and IL-12p70 secretion and decreased IL-13 levels [27].

Thus, in this clinical study, the ability of probiotic CP-9, AP-32, and ET-66 strains and heat-killed AP-32 and ET-66 strains to inhibit pathogenic bacteria in the oral cavity was further validated (Fig. 4). Viable probiotic lozenges presented better anti-pathogenic ability than heat-killed lozenges via secreting organic acids, antimicrobial peptides [28], exopolysaccharides (EPSs) [29] bacteriocins [30] (Fig. 5a and b). The functional antimicrobial components produced by viable probiotic strains should be detected by high-performance liquid chromatography (HPLC) and liquid chromatography (LC)-mass spectrometry in the future [31]. It's reported that the heat-killed probiotics possessed key advantages of viable probiotics but without the certain safety concerns of viable probiotic strains [32]. However, the antimicrobial components of heat-killed probiotics should be verified further. 


\section{Conclusion}

Mixed viable probiotic tablets, consisting of $L$. salivarius subsp. salicinius AP-32, B. animalis subsp. lactis CP-9, and L. paracasei ET-66, and heat-killed probiotics, including L. salivarius subsp. salicinius AP-32 and L. paracasei ET-66, exerted their beneficial functions by elevating the oral populations of Lactobacillus and Bifidobacterium, reducing $S$. mutans in the oral cavity, and increasing salivary IgA concentration in healthy participants. In vitro test results validated that viable probiotics effectively inhibited oral pathogens, including $S$. mutans, $P$. gingivalis, $F$. nucleatum subsp. polymorphum, and $A$. actinomycetemcomitans. Oral-nasal mucosal immunity is the frontline defense towards invading microorganisms, and this has become a crucial issue. Thus, this study will offer an alternative strategy (probiotic supplementation) to maintain oral immunity and oral health.

Acknowledgements This study was operated and funded by Bioflag Biotech Co., Ltd., Taiwan. We thank the staff of Chung Shan Medical University, Taiwan for collecting saliva samples from the participants.

Author Contributions Conceptualization: WYL, CWC, YFH, CHH, HHH; Methodology: HHH; Investigation: JHL, CRL, JFC, KCH; Formal analysis: YWK; Data acquisition and classification: JHL, CRL, JFC, KCH; Development of oral lozenges: CWC, YFH, CHH; Writing-original draft preparation: WYL; Writing—review and editing: WYL, HHH.

Funding This study was operated and funded by Bioflag Biotech Co., Ltd., Taiwan.

Data Availability The datasets used and/or analysed during the current study are available from the corresponding author on reasonable request.

Code Availability Yes.

\section{Declarations}

Conflict of interest The authors declare that they have no conflict of interest.

Consent to Participate Informed consent for participation was obtained from all participants included in this study.

\section{Consent for Publication Yes.}

Ethical Approval The protocol was approved by the Institutional Review Board of Chung Shan Medical University, Taiwan (CS19052).

Open Access This article is licensed under a Creative Commons Attribution 4.0 International License, which permits use, sharing, adaptation, distribution and reproduction in any medium or format, as long as you give appropriate credit to the original author(s) and the source, provide a link to the Creative Commons licence, and indicate if changes were made. The images or other third party material in this article are included in the article's Creative Commons licence, unless indicated otherwise in a credit line to the material. If material is not included in the article's Creative Commons licence and your intended use is not permitted by statutory regulation or exceeds the permitted use, you will need to obtain permission directly from the copyright holder. To view a copy of this licence, visit http://creativecommons.org/licenses/by/4.0/.

\section{References}

1. Takaki H, Ichimiya S, Matsumoto M, Seya T (2018) Mucosal immune response in nasal-associated lymphoid tissue upon intranasal administration by adjuvants. J Innate Immun 10(5-6):515-521

2. Wu RQ, Zhang DF, Tu E, Chen QM, Chen WJ (2014) The mucosal immune system in the oral cavity-an orchestra of $\mathrm{T}$ cell diversity. Int J Oral Sci 6(3):125-132

3. Man WH, de Steenhuijsen Piters WAA, Bogaert D (2017) The microbiota of the respiratory tract: gatekeeper to respiratory health. Nat Rev Microbiol 15(5):259-270

4. Wald ER, Guerra N, Byers C (1991) Upper respiratory tract infections in young children: duration of and frequency of complications. Pediatrics 87(2):129-133

5. Marcotte H, Lavoie MC (1998) Oral microbial ecology and the role of salivary immunoglobulin A. Microbiol Mol Biol Rev 62(1):71-109

6. Weltzin R, Traina-Dorge V, Soike K, Zhang J-Y, Mack P, Soman $\mathrm{G}$ et al (1996) Intranasal monoclonal IgA antibody to respiratory syncytial virus protects rhesus monkeys against upper and lower respiratory tract infection. J Infect Dis 174(2):256-261

7. Ruggeri FM, Johansen K, Basile G, Kraehenbuhl JP, Svensson L (1998) Antirotavirus immunoglobulin A neutralizes virus in vitro after transcytosis through epithelial cells and protects infant mice from diarrhea. J virol 72(4):2708-2714

8. Millet N, Solis NV, Swidergall M (2020) Mucosal IgA prevents commensal Candida albicans dysbiosis in the oral cavity. Front Immunol 11:2448

9. Katz J, Harmon CC, Buckner GP, Richardson GJ, Russell MW, Michalek SM (1993) Protective salivary immunoglobulin A responses against Streptococcus mutans infection after intranasal immunization with $S$. mutans antigen I/II coupled to the B subunit of cholera toxin. Infect Immun 61(5):1964-1971

10. Moutsopoulos NM, Konkel JE (2018) Tissue-specific immunity at the oral mucosal barrier. Trends Immunol 39(4):276-287

11. Lin CW, Chen YT, Ho HH, Hsieh PS, Kuo YW, Lin JH, Liu CR, Huang YF, Chen CW, Hsu CH, Lin WY, Yang SF (2021) Lozenges with probiotic strains enhance oral immune response and health. Oral Dis. https://doi.org/10.1111/odi.13854

12. Ericson D, Hamberg K, Bratthall G, Sinkiewicz-Enggren G, Ljunggren L (2013) Salivary $\operatorname{IgA}$ response to probiotic bacteria and mutans streptococci after the use of chewing gum containing Lactobacillus reuteri. Pathog Dis 68(3):82-87

13. Defrance T, Vanbervliet B, Briere F, Durand I, Rousset F, Banchereau J (1992) Interleukin 10 and transforming growth factor beta cooperate to induce anti-CD40-activated naive human B cells to secrete immunoglobulin A. J Exp Med 175(3):671-682

14. Papone V, Verolo C, Zaffaroni L, Batlle A, Capo C, Bueno L et al (2015) Detection and prevalence of periodontal pathogens in a Uruguayan population with chronic periodontitis using conventional methodology and metagenomics. Odontoestomatologia 17(25):23-32

15. Butera A, Gallo S, Maiorani C, Molino D, Chiesa A, Preda C, Scribante A (2021) Probiotic alternative to chlorhexidine in 
periodontal therapy: evaluation of clinical and microbiological parameters. Microorganisms 9(1):69

16. Wang Y, Li X, Ge T, Xiao Y, Liao Y, Cui Y et al (2016) Probiotics for prevention and treatment of respiratory tract infections in children: a systematic review and meta-analysis of randomized controlled trials. Medicine 95(31):e4509. https://doi.org/10.1097/ MD.0000000000004509

17. Laufer AS, Metlay JP, Gent JF, Fennie KP, Kong Y, Pettigrew MM (2011) Microbial communities of the upper respiratory tract and otitis media in children. mBio 2(1):e00245-10. https://doi.org/10. 1128/mBio.00245-10

18. Chen Y-T, Hsieh P-S, Ho H-H, Hsieh S-H, Kuo Y-W, Yang S-F, Lin C-W (2020) Antibacterial activity of viable and heat-killed probiotic strains against oral pathogens. Lett Appl Microbiol 70(4):310-317

19. Bonifait L, Chandad F, Grenier D (2009) Probiotics for oral health: myth or reality? J Can Dent Assoc 75(8):585-90

20. Hsieh PS, An Y, Tsai YC, Chen YC, Chuang CJ, Zeng CT, King A-E (2013) Potential of probiotic strains to modulate the inflammatory responses of epithelial and immune cells in vitro. New Microbiol 36(2):167-179

21. Elrefaei M, Burke CM, Baker CA, Jones NG, Bousheri S, Bangsberg DR, Cao H (2009) TGF- $\beta$ and IL-10 production by HIVspecific CD8+ T cells is regulated by CTLA-4 signaling on CD4+ T cells. PloS ONE 4(12): e8194

22. Ranganathan V, Akhila CH (2019) Streptococcus mutans: has it become prime perpetrator for oral manifestations. J Microbiol Exp 7(4):207-213

23. Almeida CC, Lorena SLS, Pavan CR, Akasaka HMI, Mesquita MA (2012) Beneficial effects of long-term consumption of a probiotic combination of Lactobacillus casei Shirota and Bifidobacterium breve Yakult may persist after suspension of therapy in lactose-intolerant patients. Nutr Clin Pract 27(2):247-251

24. Braathen G, Ingildsen V, Twetman S, Ericson D, Jørgensen MR (2017) Presence of Lactobacillus reuteri in saliva coincide with higher salivary IgA in young adults after intake of probiotic lozenges. Benef Microbes 8(1):17-22
25. Scharek L, Guth J, Filter M, Schmidt MFG (2007) Impact of the probiotic bacteria Enterococcus faecium NCIMB 10415 (SF68) and Bacillus cereus var. toyoi NCIMB 40112 on the development of serum IgG and faecal IgA of sows and their piglets. Arch Anim Nutr 61(4):223-234

26. Ebrahimpour-Koujan S, Milajerdi A, Larijani B, Esmaillzadeh A (2020) Effects of probiotics on salivary cytokines and immunoglobulines: a systematic review and meta-analysis on clinical trials. Sci Rep 10(1):1-11

27. Ou C-C, Lin S-L, Tsai J-J, Lin M-Y (2011) Heat-killed lactic acid bacteria enhance immunomodulatory potential by skewing the immune response toward Th1 polarization. J Food Sci 76(5):M260-M267. https://doi.org/10.1111/j.1750-3841.2011. 02161.x

28. Nair MS, Amalaradjou MA, Venkitanarayanan K (2017) Antivirulence properties of probiotics in combating microbial pathogenesis. Adv Appl Microbiol 98:1-29

29. Wu MH, Pan TM, Wu YJ, Chang SJ, Chang MS, Hu CY (2010) Exopolysaccharide activities from probiotic bifidobacterium: Immunomodulatory effects (on J774A. 1 macrophages) and antimicrobial properties. Int J Food Microbiol 144(1):104-110

30. Barefoot SF, Klaenhammer TR (1983) Detection and activity of lactacin B, a bacteriocin produced by Lactobacillus acidophilus. Appl Environ Microbiol 45(6): 1808-1815

31. Kim PI, Sohng JK, Sung C, Joo HS, Kim EM, Yamaguchi T, Kim BG (2010) Characterization and structure identification of an antimicrobial peptide, hominicin, produced by Staphylococcus hominis MBBL 2-9. Biochem Biophys Res Commun 399(2):133-138

32. Piqué N, Berlanga M, Miñana-Galbis D (2019) Health benefits of heat-killed (Tyndallized) probiotics: an overview. Int J Mol Sci 20(10):2534

Publisher's Note Springer Nature remains neutral with regard to jurisdictional claims in published maps and institutional affiliations. 